\title{
Drift mechanism of mass transfer on heterogeneous reaction in crystalline silicon substrate
}

\author{
Sergey A. Kukushkin, Andrey V. Osipov \\ Institute of Problems of Mechanical Engineering, Russian Academy of Science, \\ St Petersburg, 199178 Russia
}

This work aims to study the pressure dependence of the thickness of the epitaxial silicon carbide SiC film growing from crystalline silicon $\mathrm{Si}$ due to the heterogeneous reaction with gaseous carbon monoxide $\mathrm{CO}$ [1]. It turned out that this dependence exhibits the clear maximum (Fig. 1). On further pressure increasing the film thickness decreases [2].
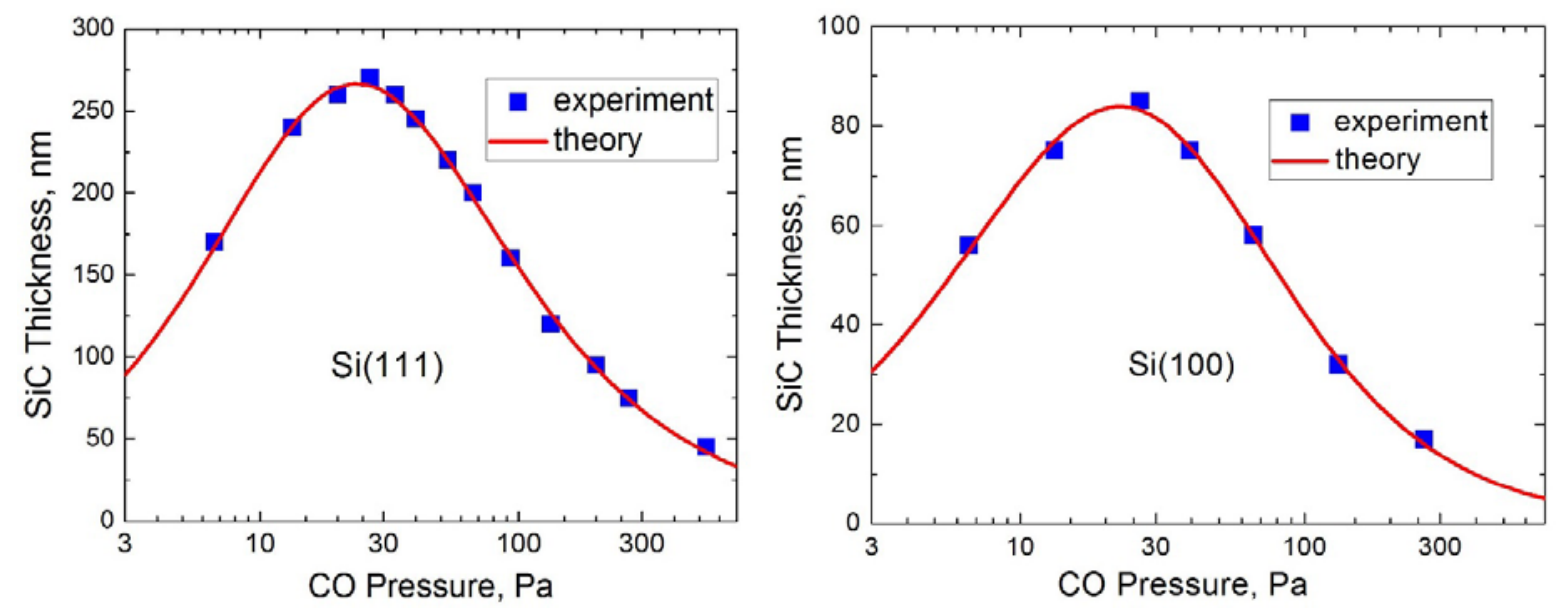

Figure 12: The measured dependences of the finite thickness of $\mathrm{SiC}$ film on $\mathrm{CO}$ pressure (squares) for $\mathrm{Si}(111)$ and $\mathrm{Si}(100)$. Solid lines are for theoretical dependences in the frames of the drift model, as obtained from Eq. (1).

As the growth mechanism, we suggest to consider the drift of molecules along the channels of the crystal which separates the gas-reagent and the zone of chemical reaction from the crystal-reagent, namely, $\mathrm{CO}$ and $\mathrm{Si}$. The theoretical model has been developed which explains such a character of the dependence by the fact that the gaseous silicon monoxide reaction product inhibits the drift of the gaseous reagent through the channels of a crystal lattice, thus decreasing their hydraulic diameter. In the proposed hydraulic model, the dependences of the film thickness both on the gas pressure and time have been calculated: 


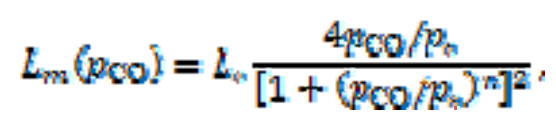

where $L_{m}$ is the maximal thickness of $\mathrm{SiC}$ film, when it is no longer growing, ${ }^{2} \mathrm{co}$ is the CO pressure, $n$ is the reversed index of polytrope, $p_{*}$ and $L_{*}$ are the parameters with dimensions of pressure and length, respectively. The dependence (1) of the film thickness on pressure contains only three parameters which could be determined from the best correspondence of formula (1) to the experimental data, using the least square method (see Fig. 1).

\section{References}

[1] S.A. Kukushkin and A.V. Osipov. Theory and practice of SiC growth on Si and its applications to wide gap semiconductor films. J. of Phys. D: Appl. Phys. 47, 313001-41 (2014).

[2] S.A. Kukushkin, A.V. Osipov: Drift mechanism of mass transfer on heterogeneous reaction in crystalline silicon substrate. Physica B, 512, 26-31 (2017). 